'Departamento de Investigación Clínica. Hospital Italiano de Buenos Aires. Ciudad de Buenos Aires, Argentina. ${ }^{2}$ Departamento de Tocoginecología, Sección Diabetes y Embarazo. Hospital Italiano Agustín Rocca, San Justo, Buenos Aires, Argentina.

${ }^{3}$ Departamento de Investigación

Clínica. Instituto Centenario. Ciudad de Buenos Aires, Argentina.

Trabajo no recibió financiamiento. Los autores declaran no tener conflictos de interés.

Recibido el 29 de enero de 2020, aceptado el 2 de julio de 2020.

Correspondencia a: Vanina Pagotto

Tte. Gral. Juan Domingo Perón 4190, C.A.B.A. Argentina. Código Postal: C1199ABB vanina.pagotto@hospitalitaliano. org.ar

\section{Evaluación de la tendencia de diabetes gestacional en un período de 11 años en Buenos Aires, Argentina}

\author{
VANINA PAGOTTO ${ }^{1}$, MARÍA LOURDES POSADAS MARTÍNEZ ${ }^{1}$, \\ DIEGO HERNÁN GIUNTA ${ }^{1}$, PABLO ANDRÉS POCHETTINO², \\ SUSANA SALZBERG ${ }^{3}$
}

\section{Trends in the frequency of obesity and gestational diabetes in an eleven years period}

Background: The prevalence of gestational diabetes (GDM) is increasing along with obesity and gestational age. This prevalence varies in populations and with different guidelines used for the diagnoses. Aim: To estimate the change in prevalence of GDM and obesity in a period 11 years. Material and Methods: Analysis of pregnancies attended at an obstetrics ward of a general hospital between 2001 and 2018. Those women who were diabetic prior to their pregnancy were excluded from the analysis. Annual crude and adjusted prevalence using direct standardization by age were estimated. Results: We analyzed 33,985 pregnancies. GDM screening was performed in 20,139 (59\%), and 1,466 (7\%) had GDM. In 2007 the crude and adjusted prevalence of GDM were 4.9 and $5.2 \%$, respectively. The figures in 2018 were 8.8 and $8.5 \%$, respectively, with an annual percentage of change (APC) of $6.9 \%(p<0.001)$. The frequency of obesity also increased with an APC of $4.1 \%(p<0.001)$. In women with GDM the APC of obesity was $4.6 \%(p<0.001)$. There was also an increase in the frequency of screening, with a joinpoint in 2011. The APC of screening in the periods 2007-2011 and 2011-2018 were 16.6 and 2.9\%, respectively $(p<0.001)$. Conclusions: The prevalence of GDM increased in this period of eleven years along with the prevalence of obesity.

(Rev Med Chile 2020; 148: 1068-1074)

Key words: Diabetes, Gestational; Mass Screening; Obesity.
S: e estima que la prevalencia de diabetes mellitus gestacional (DMG) se ha incrementado en los últimos años en forma paralela al aumento de la obesidad en el mundo y de la edad materna $^{1-4}$.

La prevalencia de DMG varía ampliamente según las diferentes publicaciones. Este hecho depende fundamentalmente de dos factores, por un lado, de las diferentes características poblacionales, y por el otro, de los distintos criterios diagnósticos utilizados por las sociedades científicas. Lo cierto es que hay diferencias metodológicas y de criterios diagnósticos en los distintos países, incluso en diferentes centros del mismo país y de la misma ciudad. En este sentido, en Latinoamérica se reportan distintas prevalencias, por ejemplo, en Colombia $2,03 \%{ }^{3}$, entre $7 \%$ y $10 \%$ en Brasil ${ }^{5}$ y en Chile $11 \%{ }^{6}$.

En Argentina se adopta la recomendación de la Asociación Latinoamericana de Diabetes (ALAD) $2007^{7}$, a la cual adhirió la Sociedad Argentina de Diabetes ${ }^{8}$ en 2008. Esta metodología fue reafirmada por un consenso de expertos de ALAD en $2016^{9}$ al incorporar los resultados del 
estudio realizado por el National Institute for Health and Care Excellence (NICE) ${ }^{10}$. En este país, la prevalencia varía entre $7,5 \%$ y $9,9 \%{ }^{11,12}$, sin embargo, existen limitadas publicaciones que hayan analizado la tendencia de la prevalencia de DMG y la frecuencia con la que se realiza el screening de este tipo de diabetes.

Es importante contar con información actualizada sobre DMG y conocer las tasas anuales por el impacto de esta entidad en la salud pública ${ }^{13,14}$. Este impacto no solo se limita a las conocidas complicaciones maternas y feto-neonatales a corto plazo, sino que la bibliografía evidencia que, tanto la madre como el hijo tienen mayor riesgo de obesidad y diabetes a largo plazo. Se estima que entre 30 y $70 \%$ de esta población desarrollará diabetes entre los 5 y 10 años posteriores a ese embarazo ${ }^{15}$. Este riesgo incrementado se debe tanto a factores genéticos, epigenéticos y conductuales, siendo estos dos últimos de radical importancia por ser modificables $^{16-19}$.

El objetivo de este estudio es estimar las prevalencias anuales de DMG, obesidad materna y también de screening de esta entidad en un período de 11 años en el Hospital Italiano de Buenos Aires, así como evaluar sus tendencias.

\section{Materiales y Métodos}

Estudio de una cohorte abierta retrospectiva de embarazadas evaluadas en el Hospital Italiano de Buenos Aires (HIBA). El protocolo fue aprobado por el Comité de Ética de Protocolos de Investigación del Hospital Italiano de Buenos Aires.

El HIBA es un sistema de salud que incluye dos hospitales universitarios y 25 centros de atención periféricos distribuidos en la Ciudad de Buenos Aires y en su periferia. El servicio de obstetricia cuenta con una guardia específica, dos pisos de internación, salas de preparto (salas de dilatantes), salas de parto y quirófanos. En el HIBA se atienden mensualmente 300 partos.

La unidad de análisis fue cada embarazo de mujeres mayores a 18 años en el período de tiempo comprendido entre 01/01/2007 y 31/12/2018. Se excluyeron los embarazos con diagnóstico de diabetes pregestacional (tanto diabetes 1 como diabetes 2).

Se definió como caso de diabetes gestacional a los embarazos con diagnóstico según las reco- mendaciones de la Asociación Latinoamericana de Diabetes (ALAD) ${ }^{9}$ : glucosa plasmática en ayunas entre 100 y $125 \mathrm{mg} / \mathrm{dl}$, valor repetido en dos determinaciones (en el curso de la misma semana); o glucosa plasmática a las $2 \mathrm{~h}$ post estímulo con $75 \mathrm{~g}$ de glucosa anhidra (p75) $\geq$ a $140 \mathrm{mg} / \mathrm{dl}$. Para cada embarazo, se recolectó la edad al inicio del mismo, la talla, el peso más cercano a la fecha de última menstruación y se clasificaron a las pacientes según su índice de masa corporal (IMC) en obesas y no obesas. Los datos fueron obtenidos de los registros electrónicos hospitalarios, tanto del listado de las pacientes evaluadas por el servicio de obstetricia como de las determinaciones de glucemia en ayuno o p75 del laboratorio. Los resultados fueron validados manualmente.

\section{Análisis estadístico}

Se estimó anualmente la prevalencia DMG cruda y ajustada, el denominador fue el número total de los embarazos y los casos fueron definidos según los criterios de la ALAD. La estandarización fue directa utilizando como población estándar a la suma de la población de todos los años. Las prevalencias fueron expresadas cada 100 embarazos y con sus intervalos de confianza de 95\% (IC95\%).

Se evaluaron las tendencias de DMG, screening y obesidad, esta última en forma global y separada en grupos con DMG y sin DMG, entre los años 2007 y 2018, utilizando un análisis de regresión joinpoint. Este análisis ajusta las líneas rectas unidas en una escala logarítmica a las tasas anuales observadas y estima el porcentual anual de cambio (APC) para cada segmento de la línea. Para este análisis, se aplicaron las configuraciones predeterminadas del software joinpoint: se permitió un máximo de 1 joinpoint para 11 años de datos. Se reportó el APC con su IC 95\% para capturar los cambios en las tendencias. Las tendencias se describieron como "crecientes" o "decrecientes" cuando el APC fue estadísticamente diferente de cero, a un alfa de 0,05 . Se consideraron "estables" las tendencias que no cumplían este criterio. Se utilizó el Joinpoint Regression Program 4.5.0.1

\section{Resultados}

En el período de estudio hubo 34.155 embarazos, se excluyeron 170 embarazos por corresponder a embarazos de mujeres con diabetes pregestacional. Los 33.985 embarazos incluidos, 
correspondieron a 27.932 mujeres, de las cuales $22.624(66,6 \%)$ eran primigestas. De las mujeres con más de un embarazo, la mediana del número de gestaciones fue 2 (intervalo intercuartílico 25\%-75\% 2-2). De los 20.139 embarazos con screening, tuvieron diagnóstico de DMG 1.466 (7,3\%) embarazos. Los 20.139 embarazos con screening representaron a 16.772 mujeres, de las cuales $15.382(91,71 \%)$ no presentaron ningún embarazo con DMG y 1.390 (8,29\%) tuvieron al menos un embarazo con DMG. De estas, 1.315 (95\%) tuvieron una único embarazo con DMG, solo $74(4 \%)$ mujeres tuvieron 2 embarazos, ambos con DMG y una mujer tuvo 3 embarazos con DMG.

\section{Diabetes mellitus gestacional}

Las prevalencias anuales de DMG, considerando como denominador solo los embarazos con screening rondaron entre $4 \%$ y $9 \%$. En la Tabla 1 se muestran las prevalencias crudas y ajustadas por año.

La tendencia de la prevalencia ajustada de DMG evidenció un incremento en la prevalencia anual con un APC de 6,9\% (IC 95\% 4,7-9,2 $\mathrm{p}<0,001)$, sin observarse ningún joinpoint. En la Figura 1 se muestra el análisis de esta tendencia.

Tabla 1. Prevalencias crudas y ajustadas de DMG expresadas cada 100 embarazos con screening

\begin{tabular}{|ccccc|}
\hline Año & Prev. crud & IC 95\% & Prev. ajust & IC 95\% \\
\hline 2007 & 4,9 & $3,6-6,6$ & 5,2 & $4,9-5,5$ \\
\hline 2008 & 4,0 & $3,0-5,4$ & 4,3 & $4,0-4,6$ \\
\hline 2009 & 5,4 & $4,3-6,8$ & 5,6 & $5,3-6,0$ \\
\hline 2010 & 5,1 & $4,1-6,3$ & 5,3 & $5,0-5,7$ \\
\hline 2011 & 4,9 & $4,0-6,0$ & 5,1 & $4,8-5,4$ \\
2012 & 6,3 & $5,2-7,7$ & 6,3 & $6,0-6,6$ \\
\hline 2013 & 6,5 & $5,4-7,9$ & 6,4 & $7,1-6,8$ \\
\hline 2014 & 8,5 & $7,3-9,9$ & 8,2 & $8,7-9,5$ \\
\hline 2015 & 9,1 & $8,0-10,3$ & 9,1 & $8,6-9,4$ \\
\hline 2016 & 9,2 & $8,1-10,5$ & 9,0 & $8,2-9,0$ \\
\hline 2017 & 8,9 & $7,8-10,2$ & 8,6 & $8,1-8,9$ \\
\hline
\end{tabular}

Prev. crud: Prevalencia cruda. Prev. ajus: Prevalencia ajustada. IC 95: Intervalos de confianzas del 95\% superior e inferior.

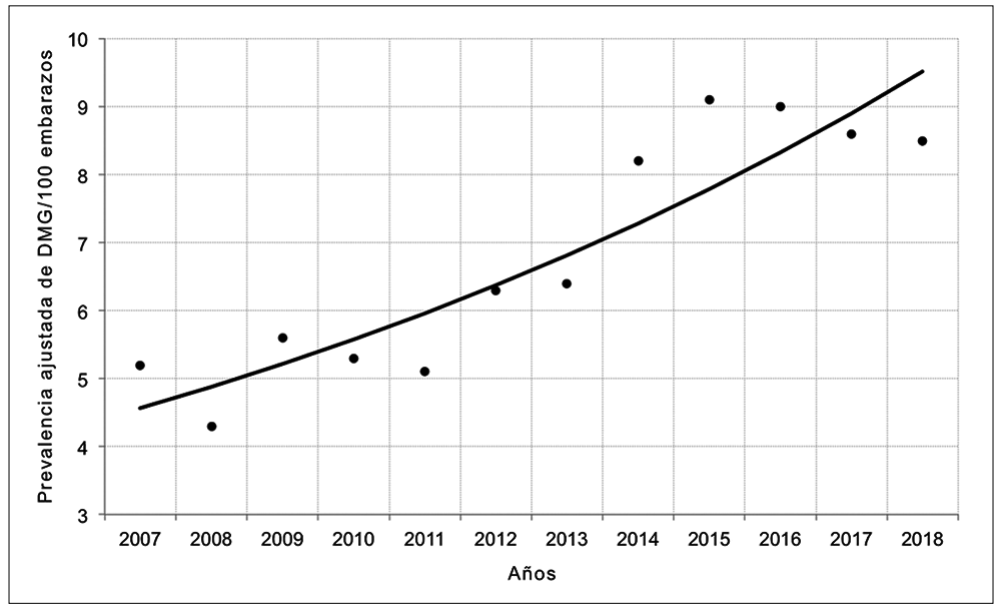

Figura 1. Tendencia de las prevalencias anuales ajustadas de DMG en el período 2007-2018. Los puntos corresponden a los valores anuales de prevalencia de DMG observados y la línea corresponde a la tendencia estimada por el modelo de DMG. La tendencia es creciente $(p<0,001)$. 


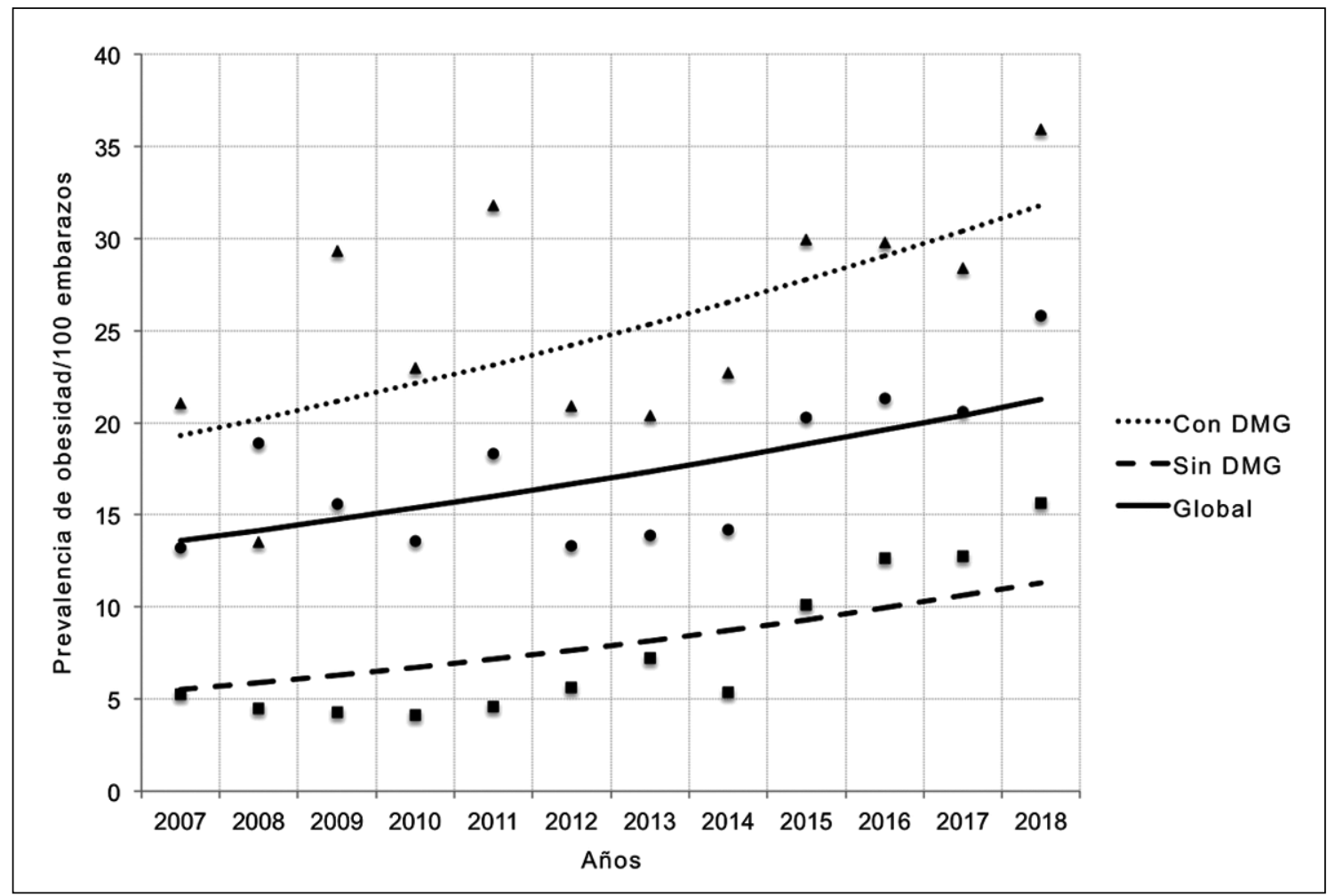

Figura 2. Tendencia de las prevalencias anuales de obesidad global y en las embarazadas con DMG y sin DMG en el período 2007-2018. Los puntos corresponden a los valores anuales de prevalencia de obesidad global observados y la línea continua corresponde a la tendencia estimada por el modelo de obesidad global. La tendencia es creciente $(p<0,001)$. Los triángulos corresponden a los valores anuales de prevalencia de obesidad en el grupo de embarazadas con DMG y la línea punteada corresponde a la tendencia estimada por el modelo de obesidad en ese grupo. La tendencia es creciente $(p<0,001)$. Los cuadrados corresponden a los valores anuales de prevalencia de obesidad en el grupo de embarazadas sin DMG y la línea con guiones corresponde a la tendencia estimada por el modelo de obesidad en ese grupo. La tendencia es estable $(p=0,2)$.

\section{Obesidad}

Considerando los embarazos con screening para DMG, no se detectó ningún joinpoint. Se observó un incremento en la prevalencia anual de obesidad global, con un APC de 4,1\% (IC 95\% 0,7$7,7 \mathrm{p}<0,001)$. Al evaluar solo los embarazos con DMG, el APC fue 4,6\% (IC 95\% 0,4-9,1 p <0,001). Por el contrario, en los embarazos sin DMG, la prevalencia de obesidad permaneció estable (APC $6,8 \%$ IC 95\% -3,9-18,7 p = 0,2). En la Figura 2 se observa el análisis de tendencias de obesidad gobal y en los embarazos con DMG y sin DMG.

\section{Screening de diabetes mellitus gestacional}

De los 33.985 embarazos, presentaron screening de DMG 20.139, lo que constituyó una prevalencia de screening de 59,3\% (IC95\% 58,7-59,8). Se observó un incremento en la prevalencia anual de screening de DMG con un joinpoint en el año 2011 (IC 95\% 2009-2013). El primer segmento entre los años 2007 y 2011 presentó un APC de $16,6 \%$ (IC95\% 12,2-21,1 p < 0,001) y el segundo segmento entre los años 2011-2018 un APC de 2,9\% (IC95\% 1,3-4,6 p < 0,001). En la Figura 3 se observa el análisis de esta tendencia.

\section{Discusión}

La DMG es un problema de salud pública creciente. Este estudio demostró que la prevalencia de DMG ha presentado un incremento sostenido desde el 2007 hasta el 2018, duplicando su valor entre el principio y fin de este período. La estan- 


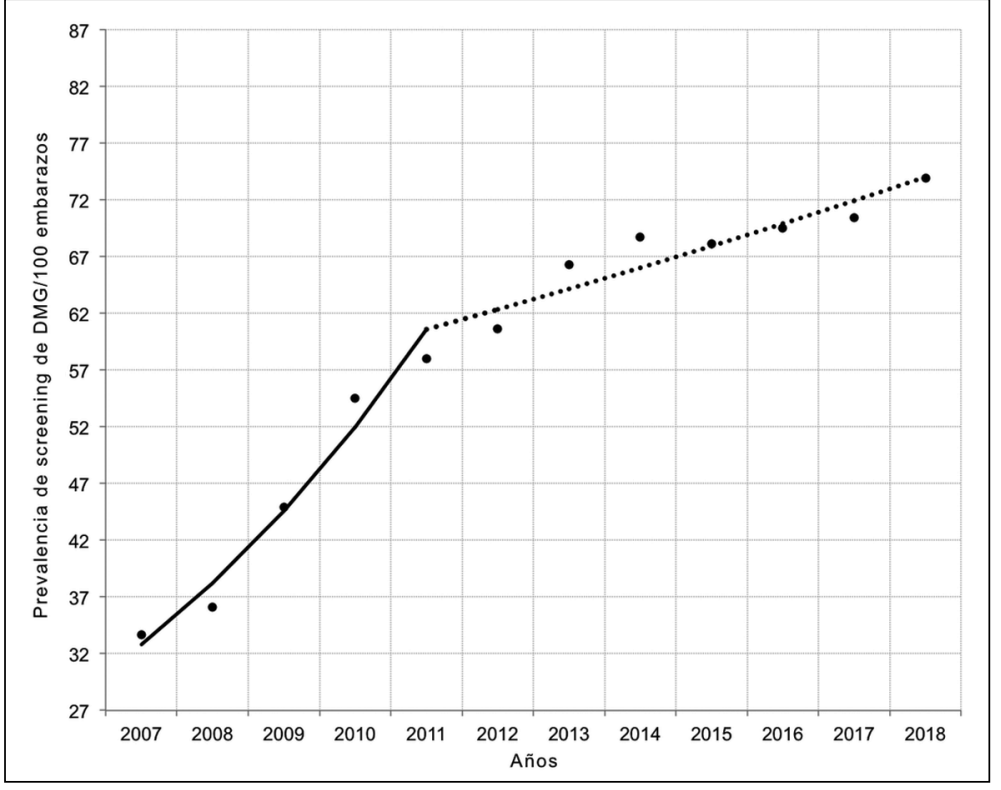

Figura 3. Tendencia de las prevalencias anuales de screening para DMG en las embarazadas en el período 2007-2018. Los puntos corresponden a los valores anuales de prevalencia de screening para DMG observados. La línea corresponde a la tendencia estimada por el modelo de screening. El punto de inflexión (joinpoint) está definido por el cambio de la línea de entera a punteada. Tendencia creciente en 2007-2011 ( $p<0,001)$ y en 2011$2018(p<0,001)$. darización por edad realizada permite inferir que muy poco de este incremento podría ser debido a cambios en la edad al momento del embarazo.

El aumento en la prevalencia de DMG podría asociarse al incremento en el screening de dicha patología que se observó a lo largo de todo el período. Otros estudios también demostraron este incremento ${ }^{20}$. Si bien se encontró un punto de inflexión en el año 2011 entre 2009 y 2013, el mismo podría deberse a múltiples causas, entre ellas, la aceptación progresiva de las guías ALAD, ya que las primeras fueron publicadas en el $2007^{7}$ y las segundas en el año $2016^{9}$.

Así mismo, se observó un aumento en la prevalencia de obesidad global y en los embarazos con DMG. En los embarazos sin DMG, la prevalencia de obesidad se mantuvo estable. Esto es concordante con varios estudios que demuestran que la obesidad es un factor de riesgo para el desarrollo de DMG. Pocos estudios han evaluado simultáneamente la tendencia de DMG y la tendencia de la obesidad en el tiempo ${ }^{21}$.

Una de las limitaciones de este estudio consiste en que la población de la que se obtuvo la muestra es la que concurrió a un hospital universitario de la Ciudad de Buenos Aires, pudiendo no ser representativa de todo el país. El sistema de salud de Argentina está compuesto por 2 sectores: público, y privado. El sector público está integrado por la red de hospitales y centros de salud públicos que prestan atención gratuita fundamentalmente a personas sin capacidad de pago. El sector privado, donde se enmarca el Hospital Italiano de Buenos Aires, asiste generalmente a personas que presentan algún sistema de cobertura de salud ${ }^{22}$. Probablemente, las embarazadas que concurren al sector privado tienen un mayor nivel socioeconómico $y$, por lo tanto, un mayor nivel educativo ${ }^{23}$, lo que las llevaría a realizar la primera consulta obstétrica a una menor edad gestacional, y a mantener una continuidad en la periodicidad de los controles, con la consiguiente mayor oportunidad de diagnóstico de DMG.

Por otro lado, las unidades de análisis fueron los episodios de embarazos. La presencia de mujeres con más de un embarazo con DMG podría impactar en el incremento en la prevalencia de DMG. Sin embargo, en esta muestra, 95\% de las mujeres tuvieron un único embarazo con DMG, haciendo poco probable el impacto mencionado.

En nuestro conocimiento, este es el primer estudio en Argentina que evaluó la tendencia de la prevalencia de DMG en un período de 11 años, teniendo como fortalezas la disponibilidad de una base de datos basada en una historia clínica electrónica que permitió la recolección de datos 
consistentes, con seguimiento a lo largo del tiempo y la realización del screening en todos los embarazos con los criterios de las guías ALAD, que son las de mayor utilización en Argentina.

El conocimiento del verdadero incremento en la prevalencia de DMG es importante para coordinar los esfuerzos requeridos para alterar esta tendencia. Esta información puede ser empleada para gestionar los recursos de salud y enfocarlos en la prevención de la obesidad, así como en el diagnóstico precoz y el tratamiento oportuno de la DMG para evitar o reducir sus complicaciones que tienen un gran impacto en la salud de la población adulta y pediátrica. Estudios similares en otros países pueden contribuir a generar una mejor visión de la situación actual.

Agradecimientos: Al Área de Gestión de la Información para la Investigación del Departamento de Investigación del Hospital Italiano de Buenos Aires por la búsqueda de los datos de los registros electrónicos, es especial a Gabriela Blugerman y a Marcela Martínez Von Scheidt.

\section{Referencias}

1. Dabelea D, Snell-Bergeon J, Hartsfield C, Bischoff K, Hamman R, McDuffie R. Increasing Prevalence of Gestational Diabetes Mellitus (GDM) Over Time and by Birth Cohort: Kaiser Permanente of Colorado GDM Screening Program. Diabetes Care 2005; 28 (3): 579-84.

2. Weinert L, Oppermann M, Salazar C, Simionato B, Silveiro S, Reichelt A. Diabetes e gestação : perfil clínico e laboratorial em pré-natal de alto risco. Rev HCPA 2010; 30 (4): 334-41.

3. Cortés Y, Ocampo T, Villegas A. Prevalence of gestational diabetes mellitus in Medellin's population from 1999 to 2000: the positive predictive value of the screening test and comparison of criteria in the NDDG and ADA. Rev Colomb Obstet Ginecol 2002; 53 (1): 81-5.

4. Voto L, Nicolotti A, Salcedo L, González M, Nasiff J, Cremonte A. Consenso de diabetes Recopilación, actualización y recomendaciones para el diagnóstico y tratamiento de la diabetes gestacional [Internet]. http:// www.fasgo.org.ar. 2012 [citado el 19 de enero de 2020]. Available from: http://www.fasgo.org.ar/archivos/consensos/diabemb.pdf

5. Do Valle J, Silva J, Oliveira Da, Martins L Lewandowski A, Horst W. Use of a clinical-laboratory score to guide treatment of gestational diabetes. Int J Gynaecol Obstet
2018; 140 (1): 47-52.

6. Huidobro A, Fulford A, Carrasco E. Incidencia de diabetes gestacional y su relación con obesidad en embarazadas chilenas. Rev Med Chile 2004; 132 (8): 931-8.

7. Márquez M, Alvariñas J, Lemay A, Faingold C, Lang J, Rodríguez B, et al. Consenso Latinoamericano de Diabetes y Embarazo. Rev ALAD [Internet]. 2007 [citado el 7 de enero de 2020]; Available from: http:// alad-americalatina.org/wp-content/uploads/2016/10/ DIABETES-Y-EMBARAZO.pdf

8. Sociedad Argentina de Diabetes [Internet]. [citado el 13 de mayo de 2020]. Available from: http://www.diabetes. org.ar/

9. Salzberg S, Alvariñas J, López G, Lapertosa L, Linari M, Falcón E, et al. Guías de diagnóstico y tratamiento de diabetes gestacional. Rev ALAD 2016; 6: 155-69.

10. National Institute for Health and Care Excellence (NICE). Diabetes in pregnancy management from preconception to the postnatal period. 2015; 5-54.

11. Shocron R, Bustos O, Nasif B, Paesani E. Prevalencia de diabetes gestacional en el Hospital Regional Río Grande-Tierra del Fuego [Internet]. Colegio de Bioquimicos de la Provincia de Cordoba. 2015 [citado el 7 de enero de 2020]. Available from: http://cobico.com.ar/ prevalencia-de-diabetes-gestacional-en-el-hospital-regional-rio-grande-tierra-del-fuego/

12. Sucani S, Maccio M, Coniberti I, Bartolin A, Zamory E, Lewin $M$, et al. Prevalence of GDM and macrosomia in an Argentinian cohort according to ALAD and IADPSG diagnosis criteria. In: Scientific Meeting of the International Association of Diabetes and Pregnancy Study Groups 2016 (IADPSG 2016) [Internet]. [citado el 7 de enero de 2020]. Available from: https://www.conicet. gov.ar/new_scp/detalle.php?keywords=\&id=30812\&congresos=yes.

13. Pinheiro R, Areia A, Mota Pinto A, Donato H. Advanced Maternal Age: Adverse Outcomes of Pregnancy, A Meta-Analysis. Acta Med Port 2019; 32 (3): 219-26.

14. Campo M, Posada G, Betancur L, Jaramillo D. Factores de riesgo para diabetes gestacional en población obstétrica en tres instituciones de Medellín, Colombia. Estudio de casos y controles. Revista Colombiana de Obstetricia y Ginecología 2012; 63 (2): 114-8.

15. Kim C, Newton KM, Knopp RH. Gestational diabetes and the incidence of type 2 diabetes: a systematic review. Diabetes Care 2002; 25 (10): 1862-8.

16. Dabelea D, Hanson R, Lindsay R, Pettitt D, Imperatore $\mathrm{G}$, Gabir $\mathrm{M}$, et al. Intrauterine exposure to diabetes conveys risks for type 2 diabetes and obesity: a study of discordant sibships. Diabetes 2000; 49 (12): 2208-11.

17. Pettitt D, Robert B, Aleck Ki Bennett P, Knowler W. 
Excessive Obesity in Offspring of Pima Indian Women with Diabetes during Pregnancy. N Engl J Med 1983; 308 (5): 242-5.

18. Silverman B, Metzger B, Cho N, Loeb C. Impaired Glucose Tolerance in Adolescent Offspring of Diabetic Mothers: Relationship to fetal hyperinsulinism. Diabetes Care 1995; 18 (5): 611-7.

19. Pettitt DJ, Aleck KA, Baird HR, Carraher MJ, Bennett $\mathrm{PH}$, Knowler WC. Congenital susceptibility to NIDDM. Role of intrauterine environment. Diabetes 1988; 37 (5): 622-8.

20. Lauwrence J, Contreras R, Chen C, Sacks, D. Trends in the Prevalence of Preexisting Diabetes and Gestational
Diabetes Mellitus Among a Racially/Ethnically Diverse Population of Pregnant Women, 1999-2005. Diabetes Care 2008; 31 (5): 899-904.

21. Ferrara A. Increasing Prevalence of Gestational Diabetes Mellitus. Diabetes Care 2007; 30 (2): 141-6.

22. Belló M, Becerril-Montekio VM. Sistema de salud de Argentina. Salud Pública Méx 2011; 53: s96-109.

23. Quintero-Medrano SM, García-Benavente D, Valle-Leal JG, López-Villegas MN, Jiménez-Mapula C. Conocimientos sobre diabetes gestacional en embarazadas de un Hospital Público del Noroeste de México. Resultados de una encuesta. Rev Chil Obstet Ginecol 2018; 83: 2506. 